\title{
La técnica de programación cepalina y los economistas en la Argentina de mediados del siglo $\mathrm{XX}^{1}$
}

\author{
Mariano Arana
}

\section{Resumen}

En este artículo, se analizan el origen y el desarrollo de la técnica de programación de Jorge Ahumada, de la Comisión Económica para América Latina (CEPAL)², y su importancia para los economistas y las instituciones programadoras del desarrollo económico. Con este fin, se estudia la circulación de la técnica en diversos organismos en la Argentina y se muestra cómo la planificación logró legitimarse en sintonía con los principales debates de la economía política de mediados del siglo XX, al tiempo que se describe el proceso de circulación institucional de los expertos y sus textos en el país. Se concluye que el aporte cepalino repercutió directamente en las instituciones locales, lo que reforzó la idea del crecimiento acelerado con estabilidad económica y contribuyó significativamente a la formación teórica de los economistas en el país.

\section{Palabras clave}

Desarrollo económico, planificación económica, CEPAL, economistas, programas de capacitación, formación profesional, historia económica, América Latina

\section{Clasificación JEL}

$$
\text { A11, B41, C61 }
$$

\section{Autor}

Mariano Arana es Licenciado en Economía y Especialista en Docencia Universitaria en Ciencias Económicas por la Universidad de Buenos Aires (UBA), Magíster en Economía Política por la Facultad Latinoamericana de Ciencias Sociales (FLACSO) e investigador y docente del Instituto de Industria de la Universidad Nacional de General Sarmiento (UNGS). Correo electrónico: marana@campus.ungs.edu.ar.

\footnotetext{
Una versión más sucinta de este trabajo se presentó en las XVI Jornadas Interescuelas/Departamentos de Historia, en la mesa temática 106: "La planificación y el Estado argentino: agencias, expertos, modelos foráneos e impacto regional (1933-1983)". Se agradecen los comentarios de Diego Pereyra, Hernán González Bollo, Aníbal Pablo Jáuregui y de los jurados revisores. Cualquier error presente en el texto es responsabilidad exclusiva del autor.

2 En virtud de la resolución 1984/67 del Consejo Económico y Social, de 27 de julio de 1984, la Comisión pasó a llamarse Comisión Económica para América Latina y el Caribe para reflejar la ampliación de su ámbito de trabajo.
} 


\section{Introducción}

A principios de la década de 1950, la experiencia planificadora en América Latina contaba con varios antecedentes. Entre estos, cabe mencionar los modos de gestión soviéticos por balances sectoriales; los ensayos estadounidenses, que se habían ocupado de estudiar los comandos agregados de la economía desde la Gran Depresión; la experiencia francesa, que se había concentrado en la inversión por su orientación hacia la modernización de los sectores productivos de forma indicativa, y, por último, los ensayos de planes quinquenales en América Latina. Los tres primeros fueron estudiados en la Comisión Económica para América Latina (CEPAL) con la finalidad de proponer un tipo diferente de planificación. El objetivo de la nueva metodología consistía en evaluar y comunicar trayectorias económicas posibles de acuerdo con la estructura existente. Se pretendió que la técnica de planificación - o de programación - elaborada en la CEPAL fuera lo suficientemente neutral para generar un sistema de decisiones que permitiera dotar de transparencia y eficacia al poder político ante la sociedad. En palabras de Celso Furtado: "Me inclinaba a pensar que el instrumento de la planificación es tan neutro como la técnica del input-output" (1988, pág. 117).

La técnica de programación fue una metodología desarrollada por la División de Desarrollo Económico de la CEPAL, dirigida por Celso Furtado e integrada por Juan Noyola, Regino Boti, Alexander Ganz y Pedro Vuscovic. Durante su primera década en la Comisión, Jorge Ahumada, quien formaba parte del equipo dirigido por Raúl Prebisch y trabajó en la comisión hasta 1961, estuvo encargado de revisar los vínculos entre la planificación y el desarrollo económico. Con sus iniciativas, planteó una combinación novedosa entre la planificación global concentrada en sectores de la producción (que se encuentra habitualmente en la matriz de insumo-producto) y la planificación de la inversión, orientada a la búsqueda de criterios de selección y evaluación de técnicas y proyectos productivos, con un nivel de agregación mayor a partir del uso de teorías de crecimiento y modelos macroeconómicos agregados. El objetivo de este trabajo es reconocer el origen y el desarrollo de dicha técnica y la importancia que tuvo para los economistas e instituciones programadoras del desarrollo económico, mediante el estudio de sus cambios en la década de 1950 y su circulación en distintas instituciones de la Argentina.

En el primer apartado, se describen los orígenes de la técnica de programación a través del análisis de los documentos iniciales. De esta manera, se identifican las influencias teóricas y los problemas que se pretendían resolver con ella, se profundiza en la imagen del economista como planificador global y se indaga sobre la evolución de estas ideas en las publicaciones de la CEPAL, al tiempo que se analizan los instrumentos técnicos y su relación con los perfiles requeridos de los economistas de la época. El siguiente apartado, previo a las conclusiones, describe, por un lado, el papel que tuvo el énfasis planificador en las instituciones de formación y los organismos de gestión pública en la Argentina y, por otro, sus vínculos con la CEPAL y la circulación de estas ideas entre economistas y funcionarios. En ambas instancias se estudia la importancia de los trabajos fundadores desde el punto de vista de la teoría y la política económicas. Se concluye que, a través de la práctica planificadora, el aporte cepalino contribuyó directamente a reforzar en las instituciones locales la idea de crecimiento acelerado con estabilidad económica y afectó significativamente la formación teórica de los economistas del país en materia de desarrollo económico. Finalmente, a la vez que se describe el proceso de circulación institucional de los expertos y sus textos en la Argentina de mediados del siglo XX, en este trabajo también se muestra cómo la planificación logró legitimarse en sintonía con los principales debates de la economía política, desde el punto de vista de la teoría y de la técnica. 


\section{La técnica de planificación y los project planners}

En el cuarto período de sesiones de la CEPAL, que se celebró en Ciudad de México en 1951, se mencionó la importancia de la asistencia técnica para el desarrollo económico en los países latinoamericanos y, con ello, surgió la necesidad de comenzar a entrenar a economistas latinoamericanos en materia de desarrollo económico. En 1952, comenzó a impartirse en Chile el Programa de Asistencia Técnica de las Naciones Unidas para el Desarrollo Económico para formar especialistas, a cargo de Jorge Ahumada. Originalmente, en los cursos se entrenaban de 12 a 14 profesionales, aunque, posteriormente, llegaron a participar más de 40 personas. El programa estaba destinado a especialistas de los centros de decisión de los distintos gobiernos de América Latina, sobre todo del ámbito ministerial. La duración del programa superaba las 250 horas de conferencias distribuidas a lo largo de tres meses, a las que se sumaban 130 horas de mesas redondas y otras actividades de investigación.

En su rol de economista de la CEPAL y de la Administración de Asistencia Técnica de las Naciones Unidas, Ahumada publicó un documento para su uso en el programa titulado "El empleo de la contabilidad del ingreso nacional en la planificación del desarrollo económico" (1952). Allí, Ahumada hacía referencia a la "acción racional" en la elección de medios y objetivos para la planificación del desarrollo, si bien reconocía la ausencia de una teoría completa sobre el crecimiento y de una metodología para planificarlo. La técnica de programación respondía al objetivo de lograr la tasa máxima de crecimiento compatible con la estabilidad y la libertad de elección del consumidor. La estabilidad se entendía como la ausencia de problemas de desempleo, inflación y balanza de pagos. Como base metodológica, citaba The Structure of American Economy, 1919-1939: An Empirical Application of Equilibrium Analysis, de Wassily Leontief (1951), obra en que se desarrollaban las técnicas de insumo-producto ${ }^{3}$, se identificaba la relevancia de estudiar la brecha entre el crecimiento potencial y real, y se revisaba el aporte de ciertos trabajos norteamericanos (como los de S. Kutnetz, E. D. Domar, Cobb-Douglas, R. Goldsmith, P. Samuelson y W. Fellner) en el uso de funciones de producción para las estimaciones de la capacidad del sistema.

Ahumada utilizó también el modelo keynesiano de Evsey Domar, que explicaba la doble función de la inversión (en cuanto efecto multiplicador, amplificador del producto y del stock de capital, y producto potencial), junto con las características de un crecimiento económico equilibrado con pleno empleo, y exponía las condiciones de estabilidad que aseguraban la igualdad entre la tasa de crecimiento del producto efectivo y la potencial. Esto permitía considerar la falta de garantías sistémicas de esas condiciones de estabilidad y crecimiento y de la necesidad de actuar sobre las funciones de inversión para acercarse a dichos objetivos. Ahumada asimiló ese peligro en su exposición, al señalar que, si la inversión provocaba un efecto multiplicador (positivo y negativo según su dirección), entonces también sería un factor desestabilizador y, por ello, habría que reducir la magnitud del multiplicador para lograr estabilidad y aumento de ahorros (Ahumada, 1952). Ahumada (1951) explicó que desarrollo equivalía al logro de la tasa máxima de crecimiento del ingreso compatible con la estabilidad en condiciones de ocupación plena. Para ello, el manejo de la inversión resultaba fundamental, ya que podría provocar inflación o desocupación, según su exceso o defecto.

La preocupación de Ahumada por el papel de la inversión en el desarrollo lo condujo a explorar criterios de selección y evaluación de proyectos de inversión. Argumentó que los criterios públicos diferían de los privados en lo referente al objeto de maximización, sobre todo porque los primeros debían tener presentes los efectos de la inversión sobre el valor agregado total; es decir, debían incluir sus resultados indirectos (hacia adelante y atrás) sobre otras esferas de la producción (Ahumada, 1955).

\footnotetext{
3 Leontief publicó la primera edición de este volumen en 1941. Aunque en los trabajos relacionados con la técnica de insumo-producto se reconocen antecedentes desde la década de 1920, es a mediados del siglo XX cuando cobran notoriedad en el ámbito académico occidental.
} 
En 1957, Ahumada participó en una conferencia sobre el desarrollo económico de América Latina celebrada por la International Economic Association (IEA) en Río de Janeiro (Brasil), que convocó a intelectuales como Ragnar Nurkse, Celso Furtado, Howard Ellis, Felipe Pazos, Gottfried Haberler, Theodore Schultz y Albert Hirschman, entre otros. Preocupado por entregar una prescripción de las prioridades de inversión que pudiera ser utilizada por los organismos financieros gubernamentales, profundizó sus críticas a los criterios neoclásicos de asignación de recursos. Señaló que estos olvidaban que el costo de oportunidad social de una mercancía era representado por su precio solo si cada factor contribuía con el mismo valor en diferentes usos productivos y sostuvo que era necesario reemplazar aquel criterio de maximización, dado que, por un lado, los diversos usos de factores arrojaban distintas contribuciones de valor y, por otro, el equilibrio era una condición excepcional y no la norma (Ahumada, 1961; Boianovsky, 2013). Las contribuciones de esta conferencia se utilizaron el año siguiente en el Manual de proyectos de desarrollo económico, en el marco del Programa CEPAL/AAT de Capacitación en materia de Desarrollo Económico.

Ahumada estima que la aplicación de la teoría ortodoxa ha conducido a preconizar aquellos criterios que implican la elevación de las utilidades al máximo, como es el caso del criterio beneficios-costos. Aunque este último se aplique corrigiendo los precios de mercado para tomar en cuenta el costo social, no por eso deja de elevar al máximo las utilidades y no puede cumplir simultáneamente las condiciones de equilibrio de la economía e igualdad de precios de los factores en todas las direcciones. (CEPAL 1958, pág. 259).

Sin embargo, el autor advertía sobre la imposibilidad de conocer el crecimiento agregado sin antes estimar la distribución de las inversiones y, al mismo tiempo, estas dependían del ritmo de la tasa de crecimiento del ingreso (Ahumada, 1955 y 1961). Por esta razón, la programación requería de un plan de inversiones sugeridas a partir del volumen de ahorros que permitiera disponer de una proyección del producto (dada la histórica relación entre capital y producto). La tarea consistía en indagar las necesidades de inversión para cumplir con el plan, estimar posibles cambios en la demanda al utilizar la técnica de elasticidad ingreso y detectar los sectores del cambio para adelantarse a las posibles fricciones y desequilibrios (escollos) en materia de desarrollo.

La técnica para compatibilizar el plan debía elegirse observando los efectos sobre todos los sectores con distintos criterios, según se requiriera cumplir objetivos de satisfacción de cantidades producidas (y necesidades manifiestas) o de eficiencia productiva. El plan debía contener pruebas de coherencia interna que evitaran la escasez de bienes y servicios, considerando las coincidencias del ahorro con la inversión, de la oferta con la demanda global y de la distribución de la inversión con los cambios esperados de la estructura de demanda.

\section{La figura del planificador global}

En la Facultad de Ciencias Económicas de la Universidad de Buenos Aires (FCE-UBA), bajo la dirección de Norberto González, se organizó en 1956 un curso sobre los problemas del desarrollo, cuyo dictado estuvo a cargo de sus profesores e investigadores, en colaboración con altos funcionarios de la CEPAL (Chirom, 1985). Según sus recuerdos de estudiante, en 1957, Mario Brodersohn (Arana, 2015b) tomó el curso intensivo de la CEPAL en la FCE-UBA junto con sus colegas Adolfo Canitrot, Bernardo Grinspun y Félix G. Elizalde. El curso - que duró tres meses - contemplaba las modalidades alternativas tanto de tiempo parcial como de tiempo completo. La mayoría de los profesores provenían de la CEPAL. Ahumada dirigía el área de economía de la Comisión y, además, participó como profesor, junto con el 
primer Ministro de Economía de la Revolución cubana, Regino Boti ${ }^{4}$. Roque Carranza ${ }^{5}$ se encargó de enseñar la matriz de insumo-producto y Raúl Prebisch dictó algunas conferencias. Posteriormente se convocó una beca para hacer un curso dirigido por Felipe Herrera ${ }^{6}$ en la sede de la CEPAL en Santiago. La beca fue aprovechada por Brodherson y Alieto Guadagni.

El año anterior a la institucionalización definitiva de los economistas en la Argentina, a partir de la creación, en 1958, de las carreras correspondientes en la Universidad de Buenos Aires (UBA), la Universidad Católica Argentina (UCA) y la Universidad Nacional del Sur (UNS), Ahumada presentó en Buenos Aires un borrador del curso "Teoría y programación del desarrollo económico" para el intensivo dictado en la FCE-UBA entre septiembre y diciembre de 19587. El texto se orientaba a la programación global mediante el estudio de técnicas que combinaban los usos de la macroeconomía de la demanda efectiva (y sus efectos multiplicador y acelerador) con los modelos de crecimiento a la Harrod-Domar, puestos en relación con las técnicas de insumo-producto (en algunos casos con usos más simples, como los modelos de sectores), en un contexto de asimetrías entre centros y periferias y heterogeneidades estructurales en las distintas regiones. La innovación no solo se daba en los énfasis y las formas de combinar el herramental existente, sino en el hecho de que se buscaba que los conceptos nuevos permitieran analizar las características diferenciales de los países de la región, pues el desarrollo no se comprendía como un proceso espontáneo ${ }^{8}$. En este sentido, es posible señalar el contenido de este entrenamiento como un modelo general para economías periféricas.

En el modelo de planificación global, se trabajaba con 10 parámetros en un sistema de 22 ecuaciones y 32 variables, con datos de dos años consecutivos. La programación global realiza el diagnóstico y fija las metas de demanda final de bienes y servicios para luego calcular la producción que debe satisfacer cada uno de los sectores, a fin de evitar estrangulamientos. El resultado se reflejó en un cuadro llamado "Presupuesto de producción y disponibilidades de bienes y servicios", que no solo combinaba la distribución de la producción entre sectores, sino también entre sus destinos en cuanto a sus usos (consumo, inversión y otros) y sus perceptores (distribución funcional del ingreso).

En sus "Notas para una teoría general de la planificación"9, Ahumada retomó el énfasis en la programación como método para aportar racionalidad a la toma de decisiones y en la neutralidad de la técnica. Para el autor, la planificación "es una técnica que sirve para elaborar políticas de una manera racional" (Ahumada, 1977, pág. 4), en la que el planificador crea las alternativas sobre las que la autoridad política decide. "La planificacion es ética y políticamente neutra" (Ahumada, 1977, pág. 4), por lo que tanto diagnosticar como planificar son tareas técnicas. En cambio, Ahumada consideraba que fijar objetivos era algo estrictamente político. En este trabajo, expresó un acercamiento discursivo

4 Además de con Ahumada y Boti, los cursos de la CEPAL en América Latina contaron con los profesores Manuel Balboa, Dudley Seers, Hollis Chenery, Ricardo Cibotti, John Galbraith, Aníbal Pinto, Adolfo Dorfman, Leopoldo Portnoy, Osvaldo Sunkel y Alberto Fracchia, entre otros (CEPAL, 1961).

5 Fue Secretario Técnico del Consejo Nacional de Desarrollo (CONADE) de la Argentina entre 1963 y 1966, donde participó en la elaboración del Plan Nacional de Desarrollo 1965-1969.

6 Ministro de Hacienda de Chile en 1953 y Director del Banco Interamericano de Desarrollo (BID) entre 1960 y 1970.

7 Este curso se dictó también en 1967, cuando se reconoció su vigencia dentro de los programas de capacitación del Instituto Latinoamericano de Planificación Económica y Social (ILPES). En 1958, la CEPAL también publicó su Manual de proyectos de desarrollo económico.

8 Entre estos conceptos nuevos, vemos los intentos de cuantificar el producto potencial y evaluar la "riqueza tangible renovable" (bienes productivos sujetos a depreciación) como unidad de densidad de capital. Además de los usos de la matriz de insumo-producto, las elasticidades precios e ingreso, la evolución de los términos de intercambio y la ecuación macroeconómica fundamental, se diferenciaron los criterios de maximización sociales de los individuales en las evaluaciones de los proyectos de inversión.

9 El texto reimpreso por el ILPES en 1977 tiene como referencia una publicación de los Cuadernos de la Sociedad Venezolana de Planificación de 1966. Sin embargo, existe una versión publicada por el Centro de Estudios del Desarrollo de la Universidad Central de Venezuela (CENDES) que data de febrero de 1962, utilizada para un seminario interno de planificación a cargo del profesor Luis Lander. En 1984, el curso dirigido por Carlos Mattos en el ILPES sobre teoría y práctica de la planificación todavía hacía referencias a dicho texto. 
a los problemas neoclásicos de escasez de recursos y maximización del bienestar, que derivaron en problemáticas de elección (de consumo y de producción, entre otras). Se encuentran allí reflexiones sobre los teoremas y axiomas referentes a la paradoja o el teorema de imposibilidad de Kenneth Arrow.

Desde sus inicios hasta 1956, el programa de capacitación de la CEPAL entrenó a 187 economistas latinamericanos, 1 de Egipto y 1 de Irán -60 en Santiago y 80 en Bogotá, en 1955 y, al año siguiente, 49 en Río de Janeiro (Brasil) - . También se realizaron programas de ocho meses de duración para especialistas becados en Santiago (CEPAL, 1957). En 1958, ya se había entrenado a 93 participantes en Santiago, el 72\% de los cuales eran economistas; el resto, ingenieros o agrónomos, y 8 provenían de la Argentina (CEPAL, 1959b). Desde 1955 hasta 1961, la CEPAL formó a 1.087 participantes en 15 cursos intensivos fuera de Chile. En la Argentina se realizaron 2 cursos con un total de 141 asistentes. El curso intensivo iniciado el 20 de septiembre de 1958 en la FCE-UBA tuvo una matrícula de 33 estudiantes a tiempo completo y 39 observadores. En 1966, el programa incluyó 1 curso básico de planificación, 4 cursos especiales (sobre educación, vivienda, salud y desarrollo económico, y planificación para dirigentes sindicales) y 10 cursos intensivos en los países de la región, en los que participaron alrededor de 700 becarios (ILPES, 2012a).

\section{Planificadores globales, sectoriales y regionales}

En 1953, la CEPAL presentó el "Estudio preliminar sobre la técnica de programación del desarrollo económico", una reflexión metodológica del Estudio Económico de América Latina y el Caribe publicado desde 1949 por la institución. Este había sido bosquejado en el cuarto período de sesiones de la Comisión, bajo el título de "Problemas teóricos y prácticos del crecimiento económico", y allí se reconocía la necesidad de adoptar programas de desarrollo y se mencionaban los elementos teóricos para su diagnóstico y las dificultades de los países periféricos. En esa instancia, se dijo que había que aprovechar los conocimientos elaborados en los países centrales para interpretar teóricamente los problemas de desarrollo económico y formar economistas en los países latinoamericanos:

Con este propósito, habría que comenzar por la realización de cursos de seminario en que los economistas de la CEPAL, con directores competentes designados al efecto, pudieran dedicar una parte de su tiempo a la elaboración de conceptos y técnicas que sirvieran para la formación de otros economistas (...). No se tiene el propósito de substituir a las múltiples escuelas o facultades de economía que existen en Latino América o competir con ellas, sino más bien de cooperar con estas instituciones, complementar su labor y trabajar con aquellos de sus egresados que, habiendo tenido experiencia en la realidad latinoamericana y responsabilidades concretas en la gestión económica, desearan especializarse en problemas de desarrollo. Se trataría, pues, de cursos de seminario para post-graduados, y, dado su índole, es de suponer que habría que trabajar en ellos con un número relativamente pequeño de personas. (CEPAL, 1952, págs. 89 y 90).

En 1955, la CEPAL publicó Análisis y Proyecciones del Desarrollo Económico, en cuya primera parte se desarrollaba la "Introducción a la técnica de programación". La técnica de programación identificaba variables comunes de los modelos keynesianos de crecimiento macroeconómico, aunque agregaba un énfasis en las relaciones económicas internacionales propio de las preocupaciones de la Comisión. Además, distinguía tempranamente los problemas de medición del volumen de capital y del uso de técnicas. Así, se refería a lo que luego se denominó la "controversia del capital" o la "controversia de Cambridge", que apuntaba a descubrir incoherencias en las proposiciones fundamentales de la teoría neoclásica del capital en su forma agregada ${ }^{10}$.

\footnotetext{
${ }^{10}$ Es probable que este texto haya contribuido al debate sobre el problema de la medición del capital, sus relaciones con la evolución del producto y la reversión de técnicas desde la periferia. Puede consultarse el trabajo de Boianovsky (2013) para profundizar en el debate teórico.
} 
Desde la perspectiva de la Comisión, la planificación debía hacerse desde lo general hacia lo particular. Estas dos fases se denominaban de proyección global y sectorial, respectivamente ${ }^{11}$, y en la primera se incluían las variables de la ecuación macroeconómica fundamental. Comenzar por las proyecciones globales permitía resolver la incógnita de las inversiones necesarias para lograr una determinada tasa de crecimiento del producto. La variable objetivo era esa tasa y desde allí se estructuraban los requisitos necesarios para cumplir el objetivo. Se argumentaba que, si se empezase por la planificación sectorial, la tasa de crecimiento no se conocería de antemano y, por lo tanto, no podría constituirse en la variable objetivo, de forma que no se cumpliría con la optimización de los esfuerzos y recursos ${ }^{12}$.

El trabajo resaltaba la neutralidad de la técnica, que permitiría discernir entre el consumo presente y futuro, así como la participación del capital extranjero y el gasto público que, según la Comisión, eran formas de financiar el crecimiento de la actividad de cara a los incrementos de un consumo futuro mayor. Al respecto se dijo:

El papel de la técnica es presentar con objetiva imparcialidad las distintas alternativas (...). Esta neutralidad de la técnica de programación no solo es recomendable para deslindar funciones, sino trambién porque fortalece la autoridad moral y el sentido persuasivo de quienes la elaboran. (CEPAL, 1955, pág. 18).

La planificación sectorial consistía en descomponer por sectores la estimación global de la demanda, estimar el crecimiento sectorial calculando las respectivas elasticidades ingreso de la demanda, de acuerdo con los incrementos proyectados, estudiar las posibilidades de sustituir importaciones y, luego, analizar la compatibilidad de cumplimiento por el lado de la oferta.

Según Ahumada (1966a), la planificación del desarrollo precisaba formar programadores globales, a los que llamó project planners. Además, se requería personal con perfil estadístico y gestor para la coordinación, debido a sus intervenciones en las distintas fases del proceso y, por último, se necesitaban programadores sectoriales: especialistas agrícolas, industriales, de transporte y de minería, entre otros. Si bien observó la necesidad de que fueran los economistas los encargados de la programación global, en el caso de los programadores sectoriales, estos debían tener un perfil con una relación más concreta con la actividad (como lo tenían los ingenieros y los agrónomos, por ejemplo). Ahumada (1966a) reconoció que los economistas de las universidades de América Latina a medidados de los sesenta no recibían una formación de ese tipo, y simpatizó con la idea de institucionalizar la profesión de programador del desarrollo.

\section{Formación y gestión con la técnica de programación}

En 1957, la FCE-UBA creó una comisión asesora para formular un nuevo plan de estudios. En la Revista de Ciencias Económicas, dicha comisión publicó dos anteproyectos para la reforma del plan: el anteproyecto del Dr. Enrique Reig y el de la Comisión de Planes de Estudio (Reig, 1958). La nueva Escuela de Economía (en la que se enmarcaban la Licenciatura en Economía y el Doctorado) incluía un seminario sobre desarrollo económico propuesto en uno de los anteproyectos, denominado

\footnotetext{
${ }^{11}$ Una vez conformadas estas dos primeras fases, quedaba por determinar tanto la política económica que debía seguirse como la organización administrativa para la ejecución. Ambas fases quedaron fuera del alcance del documento.

${ }^{12}$ Como resultado de la planificación global, se obtendrían: a) la evolución del ingreso, b) la distribución del ingreso entre consumo y ahorro, c) el monto de las inversiones necesarias y su financiación y d) la capacidad para importar y sustituir. Posteriormente, en la planificación sectorial, se conocerían: e) la demanda futura de los distintos bienes (de consumo, intermedios y de capital), f) la distribución de las inversiones necesarias, g) la demanda final y de importaciones a nivel sectorial, h) las posibilidades de exportación e i) la información sobre los niveles de productividad del trabajo.
} 
Seminario sobre Política del Desarrollo Económico ${ }^{13}$. Entre sus contenidos mínimos, se encontraba el estudio de la programación del desarrollo y evaluación de proyectos de inversión, la financiación del desarrollo económico, la programación sectorial y localización, la organización para la programación del desarrollo y la interpretación del problema del desarrollo económico argentino. El programa de ese seminario de 1963 (UBA, 1963b) cumplió con esos contenidos mínimos formulados años atrás y estuvo a cargo de Leopoldo Portnoy ${ }^{14}$. Entre sus objetivos de aprendizaje se destacaba el enfoque hacia el establecimiento de modelos, la fijación de metas y estrategias, la evaluación de los tiempos y las alternativas de inversión, la administración de proyectos, y la realización de cálculos. No solo se incluyó el programa de Ahumada, sino que se destinaron apartados exclusivamente a realizar críticas al modelo de planificación global y a estudiar problemas de planificación sectorial. En la bibliografía del seminario encontramos referencias tanto a la econometría (L. Klein, F. Toranzos y R. G. D. Allen) como a la programación del desarrollo (J. Ahumada, J. Tinbergen, A. Hirschman, Naciones Unidas y $\mathrm{H}$. Chenery) y a otros autores relacionados con los procesos de acumulación de capital (R. Nurkse y N. Kaldor). En 1967, se actualizó parte de los contenidos del seminario, incluyendo la programación del desarrollo económico, concentrada en autores como Arthur Lewis, Walt W. Rostow y Jan Tinbergen, entre otros (UBA, 1967). No fue hasta 1973 cuando la asignatura "Desarrollo económico" comenzó a tener una impronta dependentista, alejada de las preocupaciones por la medición del desarrollo propias del contexto político y universitario (UBA, 1973).

En 1960, el Consejo Federal de Inversiones (CFI) organizó el Curso Intensivo de Capacitación en Problemas de Desarrollo Económico. Bajo la autoría de Héctor Grupe y Oscar Cornblit, se publicó un apartado sobre programación regional que incluía temáticas propias de sus áreas de competencia (transportes, ubicación, aglomeración y estudios agrarios, entre otros). Alfredo Eric Calcagno (Arana, 2018) relata no solo haber tomado el curso de la CEPAL como estudiante en la FCE-UBA, sino también haber viajado a Chile, cuando estuvo a cargo del CFI, con el objetivo de pedir autorización a Prebisch para dictarlo en la institución argentina. Prebisch accedió, y también ofreció colaborar con los materiales y con la participación de algunos docentes de la CEPAL. Así, en 1960, el curso intensivo se dictó en el Departamento de Graduados de la FCE-UBA ${ }^{15}$. El temario incluyó la elaboración de herramientas para la programación del desarrollo económico, para lo cual se utilizó principalmente el texto redactado por Jorge Ahumada en 1959 para el curso de la UBA (Cuello y Tandeciarz, 1964). Se mostraron distintos modelos de programación para economías cerradas y abiertas, sobre la base de elementos de la macroeconomía, combinados con estimaciones sectoriales de las cuentas nacionales (de los sectores agropecuario, industrial y de servicios) e instrumentos clásicos de las teorías del crecimiento (relaciones capital-producto, tasas de depreciación y stock de capital). También se agregaron elementos no tradicionales de la modelización, como los coeficientes de los términos de intercambio e indicadores de remisión de utilidades. La planificación consistía en prescripciones sencillas respecto del diagnóstico de las variables recopiladas y la fijación de objetivos probables y deseables, para luego revisar la distribución del crecimiento compatible con los componentes de la demanda agregada y los sectores de la producción. De esta manera, la programación arrojaba como resultado el presupuesto nacional,

\footnotetext{
${ }^{13} \mathrm{Si}$ bien algunos temas del desarrollo económico se encontraban en áreas del plan vigente (el "plan D" de su Seminario sobre Política del Desarrollo Económico), el nuevo plan no reconocía equivalencias con su precedente (CECE, 1962). En el programa del tercer curso de la asignatura "Economía" de 1958 (todavía en el plan D) de la FCE-UBA, se ampliaron las temáticas de la unidad sobre desarrollo económico para incluir "La programación de desarrollo", "Los planes de corto y largo plazo" y "El balance de pagos y las inversiones extranjeras". En los anteproyectos de reforma del plan de estudios de 1958, figuraba la asignatura "Seminario sobre desarrollo económico y estructura económica contemporánea".

${ }^{14}$ Portnoy (1918-1997) fue contador público y Doctor en Ciencias Económicas por la UBA, donde se desempeñó como docente y decano. Fue docente del programa de la CEPAL dictado en la FCE-UBA.

${ }^{15}$ Al asumir Álvaro Alsogaray el Ministerio de Economía de la Nación en 1959, Mario Brodershon se trasladó a la provincia de Buenos Aires, donde Aldo Ferrer era Ministro de Economía y Norberto González dirigía la Junta de Planificación Económica. González utilizó el curso elaborado por la CEPAL y dictó programación económica, y Brodershon introdujo los trabajos prácticos. Ambos replicaron este esquema en los cursos de graduados de la FCE-UBA en 1960, donde estudiaron Juan C. Gómez Sabaíni y Raúl Cuello (Arana, 2015b).
} 
que significaba, en el plano agregado, lo que el presupuesto fiscal significaba para las proyecciones e intenciones del sector público. El mismo curso fue dictado en el marco del Departamento de Graduados en 1963 por Carlos A. García Turdero, y tuvo continuidad al menos durante los dos años siguientes ${ }^{16}$.

\section{Objetividad, neutralidad y control}

En diciembre de 1959, se otorgó carácter académico al Instituto de Investigaciones de Estadística y Matemática Aplicada que se había creado dos años antes en la FCE-UBA. Algunos de los títulos más representativos del trabajo realizado en el Instituto son "Modelo de crecimiento económico del tipo lineal-logarítmico con tasa evolutiva", "Metodología para el modelo sectorial de crecimiento para la economía argentina" y "Sobre la cuantificación del progreso tecnológico en un país en desarrollo", junto con el seminario Modelos de Expansión Económica Equilibrada. Otro modelo de programación económica por sectores fue elaborado por Carlos Eugenio Dieulefait (1958) ${ }^{17}$. En su trabajo, utilizó los aportes de Leontief para realizar un modelo matemático que integrara todos los sectores nacionales por medio del uso de las recomendaciones metodológicas de la CEPAL para ahorrar costos del uso de maquinaria electrónica de cálculo. Así, trató de fijar metas de demandas finales para conocer los requisitos intermedios de cada uno de los sectores. El artículo contribuyó a difundir técnicas de programación modernas asociadas a la idea de control del proceso productivo.

En la Revista de Ciencias Económicas se publicaron varios modelos matemáticos relacionados con los problemas del crecimiento y la distribución, como el "modelo lineal de expansión económica equilibrada", de Fausto Toranzos (1962) ${ }^{18}$, que, a partir de relaciones expresadas en la matriz de Leontief, señaló que el problema de la planificación del desarrollo equivalía a determinar la expansión de la demanda final que provocara el mayor crecimiento del producto nacional sujeto a las condiciones de oferta de los factores de producción. De este modo, el problema del proceso de desarrollo consistía en maximizar el producto nacional dentro de los recursos disponibles y, por lo tanto, constituía un problema de programación lineal.

En diciembre de 1961, el Colegio de Graduados en Ciencias Económicas presentó un ciclo de conferencias sobre la situación económica del país, donde participaron Carlos Moyano Llerena, Francisco García Olano, Manuel San Miguel, Leopoldo Portnoy, Federico Pinedo y Adalbert Krieger Vasena. San Miguel y otros (1961) sugirieron que los desequilibrios estructurales no se resolverían con políticas de estabilización. En su lugar, señalaron que el desarrollo armónico y acelerado mediante el uso de las técnicas de programación permitiría acelerar el crecimiento, mejorar la productividad, incrementar el capital social básico (transporte, comunicaciones y energía), mejorar la organización de las empresas y, con ello, morigerar los efectos propagadores de la inflación (precio y salario), para así revertir los efectos depresivos de la caída de los términos de intercambio.

La idea de control del proceso de desarrollo fue transversal a casi todas las intervenciones de este ciclo de conferencias. En esa época, el inventario de los economistas incluía las técnicas de programación lineal, el cálculo de coeficientes técnicos y de elasticidades de las demandas, la evaluación de los efectos sobre la balanza de pagos, el cálculo de la necesidad de financiación externa y la estimación de los costos de los principales proyectos de inversión. Según San Miguel y otros (1961), estas eran de "carácter eminentemente neutral".

\footnotetext{
${ }^{16}$ La FCE-UBA cuenta con publicaciones de 1964 sobre temas de contabilidad pública y matriz de insumo-producto elaboradas por el Departamento, y el curso se reimprimió al año siguiente (UBA, 1964 y 1965).

17 Dieulefait, nacido en 1901, fue Director Nacional de Investigaciones Estadísticas y Censos en 1946.

18 Toranzos nació en 1908, se doctoró en Ciencias Fisicomatemáticas y participó en la Comisión del Plan de Estudios que se encargó de la creación de la carrera en 1958. Fue titular de la cátedra de Estadística en la FCE-UBA.
} 


\section{Los usos de la técnica de programación}

En 1956, Manuel Balboa y Alberto Fracchia trabajaron en un documento titulado "El capital fijo renovable de la República Argentina en el período 1935-1955", que sería presentado al año siguiente en la Quinta Conferencia General de la Asociación Internacional de Investigaciones sobre Rentas y Riqueza, en Arnhem (Países Bajos), y publicado en 1959 por Desarrollo Económico: Revista de Ciencias Sociales (Balboa y Fracchia, 1959) ${ }^{19}$. Dedicado a estudiar temas de contabilidad social, en 1957 Balboa incluyó el estudio de los "esquemas generales de las cuentas sociales y modelos económicos para la totalidad de la economía" (Besa García, 1992, pág. 3) en sus clases de estadística económica en Santiago. Al año siguiente publicó "La utilización del modelo de insumo-producto en las proyecciones de la economía argentina" en Desarrollo Económico: Revista de Ciencias Sociales (Balboa, 1958), trabajo que sería utilizado ese mismo año en uno de los textos más importantes de la época: "El desarrollo económico de la Argentina”, publicado por la CEPAL (1959a) como parte de su serie Análisis y Proyecciones del Desarrollo Económico, comenzada en 1955. De hecho, su tomo inicial fue, precisamente, "Introducción a la técnica de programación". En el trabajo se incluyeron datos sobre 23 sectores productivos, y se hizo hincapié en la explicación de la dinámica de las importaciones y la integración de los planes sectoriales en la programación global (CEPAL, 1959a). Los aportes de Balboa en el marco de la CEPAL continuaron a lo largo de la década de 1960, orientados a la aplicación de estas técnicas en diversos países y a la producción de material de apoyo para los cursos en los que participó (Besa García, 1992). En lo que respecta al fomento de políticas orientadas a la sustitución de importaciones, Balboa presentó en Río de Janeiro (Brasil) un trabajo basado en este estudio en la Conferencia Regional para la Investigación de la Renta y la Riqueza, proyectando tres hipótesis de crecimiento para el período 1962-1967. La técnica de programación, junto con el modelo de insumo-producto, sirvieron para analizar la estructura intersectorial y las consecuencias de las proyecciones de la demanda final (Balboa, 1960).

Un año después de su fundación en 1962, el Consejo Nacional de Desarrollo de la Argentina (CONADE) y Ahumada figuraban como los autores de la publicación del curso sobre teoría y programación del desarrollo. Este fue uno de los primeros registros de los entrenamientos impartidos en la institución, que había sido creada en 1961 durante el gobierno de Arturo Frondizi. El CONADE trabajó en conjunto con varias instituciones locales y externas. El programa de colaboración con la CEPAL sobre cuentas nacionales lo dirigió Alberto Fracchia, mientras que Federico Herschel condujo otro sobre tributación. En la creación de la institución figuraron como consejeros Manuel San Miguel y Carlos Eugenio Dieulefait, entre otros. San Miguel logró continuidad cuando se reestructuró el organismo durante el gobierno de Arturo Illia, ya que el nuevo Secretario Ejecutivo pasó a ser Roque Carranza, secundado por Bernardo Grinspun (Jáuregui, 2013); todos ellos estaban vinculados a la FCE-UBA y habían participado, en algunos casos, en la elaboración de los artículos, y en otros, en los entrenamientos sobre planificación del desarrollo realizados pocos años antes. No es extraño, como señala Jáuregui (2014), que Roque Carranza quisiera darle un enfoque técnico a la gestión económica, que, al contrario del sesgo frondizista, se acercara a las "ideas de crecimiento equilibrado" (pág. 150).

Aunque hubo continuidad en lo que respecta a los funcionarios que participaron, De Pablo (1995, pág. 147) resalta que Carranza no hizo mención alguna de la utilización de la matriz insumo-producto en las proyecciones globales del Plan Nacional de Desarrollo 1965-1969. Asimismo, indica que tampoco se utilizaron los documentos elaborados durante la gestión de San Miguel. En el CONADE, en 1962,

\footnotetext{
${ }^{19}$ En 1952, Balboa fue asesor principal del entonces Ministerio de Asuntos Económicos, dirigido por Alfredo Gómez Morales. En 1955, participó en el grupo conjunto del Gobierno argentino y las Naciones Unidas para la programación del desarrollo económico. Elaboró los volúmenes de Contabilidad social publicados por el ILPES en 1963 ([en línea] https://repositorio.cepal. org/handle/11362/33203), que se utilizaban desde 1961 en el Programa Conjunto CEPAL/DOAT de Capacitación en materia de Desarrollo Económico. Por su parte, Fracchia trabajó en el Banco Central de la República Argentina (BCRA), el CFI, el CONADE y el Instituto Nacional de Estadística y Censos (INDEC). Fue profesor de la UBA y participó en el programa de capacitación de la CEPAL como profesor de Contabilidad Social. Ambos son considerados los iniciadores de las cuentas nacionales en la Argentina.
} 
la tecnología utilizada por los calculistas se basaba en hojas de cálculo rectangulares y máquinas de calcular manuales (Facit y Olivetti) y eléctricas (Friden y Olivetti). Una regresión simple de 30 observaciones demoraba varias horas, sin posibilidad de verificar errores de cálculo de manera sencilla. Recuerda este economista que esa tabla "terriblemente impactante" (la matriz de insumo-producto de 1953) era una hoja enorme pegada en la pared, cuyos cálculos de requisitos intermedios eran realizados por Clementina, la computadora modelo Mercury que funcionaba en la Facultad de Ciencias Exactas y Naturales de la UBA desde $1961^{20}$.

En la primera reunión anual de la Asociación Argentina de Economía Política (AAEP), en la ciudad de Río Tercero en 1964, los integrantes del CONADE, Julio Berlinski, Faustino González, Clemente Panzone y Jacobo Rabinovich ${ }^{21}$, publicaron un documento sobre el método de proyecciones seguido en el marco del plan de desarrollo a cargo de esa institución (Berlinsky y otros, 1964). En el trabajo se mostraba una secuencia de planificación similar a las de la técnica de programación. En primer lugar, se realizaban las proyecciones macroeconómicas mediante un modelo de 7 ecuaciones con 16 variables y parámetros, cuyo objetivo era fijar la tasa de crecimiento del producto y analizar los requisitos de inversión y del sector externo que posibilitaran cumplir con la meta. Posteriormente, se pasó a analizar las posibilidades de producción de los sectores, para lo que se recortaba una muestra de 11 sectores de relevancia dentro de la industria, el transporte y la energía (el resto de los sectores no contaba con información sistemática y resultaban problemáticos a la hora de modelizar). El trabajo dejaba entrever la atención particular prestada al sector externo y a la sustitución de importaciones evidenciada en todos los documentos sobre la técnica de programación y, en consecuencia, no hacía referencia al cruce de las estimaciones reales con la esfera financiera ${ }^{22}$.

En 1967, el Centro de Investigaciones Económicas del Instituto Torcuato Di Tella (ITDT) publicó su documento de trabajo número 39, titulado "Utilización del modelo de insumo-producto como instrumento de proyección en la Argentina" (Brodersohn y Guisarri, 1968), donde se evaluaban las capacidades de cuatro técnicas de proyección sobre la actividad económica: las técnicas basadas en la matriz de insumo-producto (en sus versiones de 1953, 1960 y 1963), la regresión múltiple, la expansión del producto bruto interno y la expansión de la demanda final. Mediante dicha investigación, se concluyó que las primeras dos técnicas lograban márgenes de error de predicción muy inferiores a las últimas. En el caso argentino, la regresión múltiple tenía la ventaja de suponer costos de elaboración mucho menores y lograr mejores estimaciones globales. Sin embargo, paradójicamente, los resultados de la técnica de insumo-producto eran más acertados cuanto mayor era el período de proyección. El trabajo era una ampliación del ejercicio de sensibilidad de los coeficientes de la matriz de insumo-producto de 1960 realizado por el CONADE, en el que se había concluido que, pese a las debilidades de la técnica, su uso para proyectar resultaba confiable (Brodersohn y Guisarri, 1968). Como sus colegas, los técnicos del CONADE Osvaldo Fernández Balmaceda, Reinaldo Félix Bajraj, Guillermo Calvo y Julio Alberto Piekarz presentaron sus investigaciones en las primeras jornadas de la AAEP de 1964, las cuales fueron publicadas al año siguiente en Desarrollo Económico: Revista de Ciencias Sociales.

\footnotetext{
20 Para terminar su tesis de maestría en Harvard, y ayudado por personal del Instituto de Cálculo y un matemático, Mario Brodersohn pidió turno para usar a Clementina y tuvo que esperar tres meses para invertir una matriz de insumo-producto de 20 filas por 20 columnas. En los sesenta, en el Instituto de Cálculo trabajaron economistas matemáticos como Arturo O'Connell y Roberto Frenkel.

${ }^{21}$ Publicaron su trabajo al año siguiente en Desarrollo Económico: Revista de Ciencias Sociales. Según recuerda De Pablo (1995), Faustino González estaba a cargo del sector de programación global, en el que trabajaban los mejores profesionales del CONADE.

22 Aunque los cursos intensivos tenían módulos sobre política monetaria, es notable la ausencia de referencias a movimientos de capitales y tasas de interés en toda la bibliografía revisada.
} 


\section{Conclusiones}

En este trabajo se indagó sobre el desarrollo y la importancia de la técnica de programación, destinada a economistas e instituciones programadoras del desarrollo económico, durante las dos décadas que siguieron a sus orígenes en 1952. Se estudiaron sus principales transformaciones y su circulación en distintas instituciones de la Argentina, en un contexto en que se estaban produciendo en la región grandes cambios en el ámbito de la planificación indicativa y los idearios.

En primer lugar, hay que notar el hincapié que se hacía en el logro del crecimiento planificado, acelerado y estable, que introdujo estos documentos en los debates de fines de los años cincuenta sobre crecimiento equilibrado y desequilibrado, encabezados por Ragnar Nurkse y Albert Hirschman, respectivamente. Este enfoque también estaba en sintonía con el uso de nuevas técnicas y tecnologías de cálculo. La programación lineal, las calculadoras electrónicas y las computadoras (principalmente para el uso sobre matrices de insumo-producto), junto con la posibilidad de explotar datos antiguos con nuevas herramientas y de construir otras interpretaciones a partir de ellas, alimentaron la confianza de los expertos en la capacidad de conducir el proceso económico de manera racional. Esta racionalidad se expresó en el uso de técnicas "neutrales", que evitaban el desvío de la objetividad del investigador, de manera que permitían creer que, si los resultados estaban bien calculados, se podrían cumplir las metas planteadas. El experto debía ofrecer diversos caminos en el plano económico para cumplir con las metas sociales seleccionadas por los políticos.

Si bien, al principio, la técnica se basó solo en la práctica y se reconoció una escasez teórica, a medida que pasaron los años, la búsqueda de definiciones conceptuales y de perfeccionamiento del método hicieron que, en el caso de Ahumada, se reflejase el esbozo de una teoría general. Las ideas de reconocidos economistas extranjeros como John M. Keynes y Wassily Leontief aparecieron frecuentemente en los escritos sobre planificación: por un lado, las herramientas teóricas keynesianas, en ese entonces vinculadas a la ortodoxia en los países anglosajones, y, por otro, los instrumentos técnicos de Leontief, que ampliaban la macroeconomía keynesiana y permitían aplicarla tanto a los países centrales como a los periféricos. Cuando Ahumada intentó extrapolar su práctica planificadora hacia un modelo general de economías latinoamericanas, también hizo uso de Arrow, un teórico del equilibrio general y uno de los economistas más destacados de la teoría neoclásica (Ahumada, 1977). La planificación general de los controles centrales macroeconómicos, la planificación mesoeconómica de la matriz de insumo-producto, la selección de técnicas y proyectos de inversión, y el gobierno de nuevos modelos de ecuaciones contribuyeron a precisar los cálculos, afinar la puntería y depositar mayor confianza en la técnica. Si bien Jorge Ahumada, como economista de la CEPAL, dialogó con esos textos centrales en la economía política occidental y tuvo pretensiones creativas al respecto, no parece habérsele reservado un espacio relevante en los discursos sobre la modernización mediante la planificación del desarrollo o, incluso, en el pensamiento económico latinoamericano. No obstante, el impacto que tuvo en su momento parece ser lo suficientemente significativo para permitirle figurar en la agenda de investigación social del pasado económico.

Además de destacar las contribuciones y referencias teóricas de Ahumada - su capacidad creativa, su inserción ideológica y sus usos técnicos - , el segundo aspecto de esta historia hace referencia a su rol en el contexto institucional. Por un lado, la CEPAL, a través de estos cursos, pretendió complementar la formación que recibían los economistas en las universidades y los organismos públicos, fomentó el entrenamiento de programadores globales y por sectores productivos, concedió becas a especialistas de centros de decisión y estableció diálogos fluidos con autoridades de gobierno, universidades y expertos de toda América Latina.

En el caso particular de la Argentina, los entrenamientos para la planificación circularon en cursos, materias y planes de estudios de la UBA-FCE, en asociaciones profesionales (la AAEP y el Departamento 
de Graduados), en editoriales (la Editorial Universitaria de Buenos Aires (EUDEBA) y la CEPAL), en el gobierno nacional (el CONADE y los ministerios) y en el provincial (el CFI) y en revistas especializadas (Revista de Ciencias Económicas y Desarrollo Económico: Revista de Ciencias Sociales). De esta forma, influyeron en las reformas de los planes de estudio de los economistas, al tiempo que actuaron como instituciones de formación de posgrado en una región donde prácticamente no existían ese tipo de especializaciones. Ante la ausencia total de programadores del desarrollo, la CEPAL desarrolló diversos entrenamientos. Toda esta amplitud temática provocó debates, investigaciones, publicaciones, empleos y políticas. El entrenamiento de especialistas y la contribución a los planes de desarrollo parecen ser sus dos mayores contribuciones.

Para 1972, los problemas sociales y políticos asociados a la planificación ya se hacían evidentes, y el mismo ILPES reconocía un exceso de confianza en los planes de desarrollo (ILPES, 2012b). Ni la teoría ni la técnica planificadora fueron objeto de cuestionamientos, sino las dificultades impuestas por los procesos políticos. A partir de entonces, se hizo hincapié en adaptar los mecanismos de planificación a las circunstancias particulares de cada país, pero se sostuvo su importancia para el sistema técnico-administrativo del Estado. Estas configuraciones de técnicas, teorías e instrumentos se combinaron con la circulación de especialistas en instituciones clave de formación de economistas y de políticas públicas en la Argentina, lo que propagó las ideas y prácticas contenidas en los textos de la CEPAL sobre la planificación del desarrollo en América Latina. De este modo, no solo se amplió el debate de ideas, sino que se contribuyó a obtener nueva información para el estudio económico de la región.

\section{Bibliografía}

Ahumada, J. (1977), “Notas para una teoría general de la planificación”, Documentos de Proyectos, Estudios e Investigaciones (INT-0173), Santiago, Instituto Latinoamericano y del Caribe de Planificación Económica y Social (ILPES).

(1967), "Teoría y programación del desarrollo económico", Cuadernos del ILPES, vol. 1, № 1, Santiago, Instituto Latinoamericano y del Caribe de Planificación Económica y Social (ILPES).

(1966a), "Necesidades de formación del personal de las instituciones para el desarrollo económico", Revista Mexicana de Sociología, vol. 28, № 1.

(1966b), "Notas para una teoría general de la planificación", Cuadernos de la Sociedad Venezolana de Planificación, vol. 4, № 4-5, Caracas.

(1962), "Teoría y programación del desarrollo", Buenos Aires, Consejo Nacional de Desarrollo (CONADE). (1961), "Investment priorities", Economic Development for Latin America, H. Ellis y H. Wallich (eds.), Londres, Palgrave Macmillan.

(1959), "Discurso del doctor Jorge Ahumada en el acto de clausura del Curso Intensivo de Capacitación en Problemas de Desarrollo Económico", Revista de Desarrollo Económico, vol. 2, № 2.

(1958), Teoría y programación del desarrollo económico, Buenos Aires, Universidad de Buenos Aires (UBA)/Comisión Económica para América Latina (CEPAL).

(1955), "Preparación y evaluación de proyectos de desarrollo económico", El Trimestre Económico, vol. 22, No 87.

(1952), "El empleo de la contabilidad del ingreso nacional en la planificación del desarrollo económico", Documentos de Proyectos, Estudios e Investigaciones (INT-1749), Santiago, Comisión Económica para América Latina (CEPAL), noviembre.

(1951), "Desarrollo económico y estabilidad", El Trimestre Económico, vol. 18, № 71.

Arana, M. (2018), "Entrevista a Alfredo Eric Calcagno", octubre, inédito.

(2015a), "En el núcleo de la edad dorada del desarrollo: la Revista de Ciencias Económicas, UBA (1958-1963)", tesis de maestría en economía política, Buenos Aires, Facultad Latinoamericana de Ciencias Sociales (FLACSO).

(2015b), "Entrevista a Brodersohn, Mario S.", mayo, inédito. 
(2013), "Entrevista a Arturo O'Connell", julio, inédito.

Balboa, M. (1963), "Construction and use of input-output tables in Latin American countries", Structural Interdependence and Economic Development, T. Barna (ed.), Londres, Palgrave Macmillan.

(1960), "Algunas aplicaciones del modelo de insumo-producto al análisis y a las proyecciones de la economía argentina", Boletín Económico de América Latina, vol. 5, № 1, Santiago, Comisión Económica para América Latina (CEPAL).

(1958), "La utilización del modelo de insumo-producto en las proyecciones de la economía argentina", Desarrollo Económico: Revista de Ciencias Sociales, vol. 1, № 1.

Balboa, M. y A. Fracchia (1959), "El capital fijo renovable de la República Argentina en el período 1935-1955", Desarrollo Económico: Revista de Ciencias Sociales, vol. 2, № 2.

Berlinski, J. y otros (1964), "Metodología de las proyecciones del plan de desarrollo" [en línea] https://aaep. org.ar/anales/works/works1964/Berlinski.pdf.

Besa García, J. (1992), "Escritos de Manuel Balboa 1952-1976: bibliografía existente en la Biblioteca de la CEPAL" [en línea] https://repositorio.cepal.org/bitstream/handle/11362/3400/FILE\%20\%28147914\%29_ es. pdf? sequence $=1$ \&isAllowed=y.

Boianovsky, M. (2013), "The Economic Commission for Latin America and the 1950s' debate on choice of techniques", Review of Political Economy, vol. 25, № 3.

Brodersohn, M. y A. Guisarri (1968), "Utilización del modelo de insumo-producto como instrumento de proyección en la Argentina", El Trimestre Económico, vol. 35, № 140.

CECE (Centro de Estudiantes de Ciencias Económicas) (1962), "Guía del estudiante", Buenos Aires.

CEPAL (Comisión Económica para América Latina) (1961), Informe sobre el Programa Conjunto CEPAL DOAT de Capacitación en Materia de Desarrollo Económico (E/CN.12/588), Santiago, marzo. (1959a), "El desarrollo económico de la Argentina", Análisis y Proyecciones del Desarrollo Económico, vol. 5 (E/CN.12/429/Add.3/Rev.1), Ciudad de México, agosto.

(1959b), Informe sobre el Programa Conjunto CEPAL/AAT de Capacitación en Materia de Desarrollo Económico (E/CN.12/523), Santiago, marzo.

(1958), Manual de proyectos de desarrollo económico (E/CN.12/426/Add.1/Rev.1), Ciudad de México, diciembre.

(1957), Informe acerca del Programa Conjunto CEPAL/AAT sobre Capacitación de Economistas en Desarrollo Económico (E/CN.12/433), Santiago, abril.

(1955), "Introducción a la técnica de programación", Análisis y Proyecciones del Desarrollo Económico, vol. 1 (E/CN.12/363), Ciudad de México, julio.

(1953), Estudio preliminar sobre la técnica de programación del desarrollo económico (E/CN.12/292), Santiago, marzo.

(1952), Problemas teóricos y prácticos del crecimiento económico (E/CN.12/221), Ciudad de México, septiembre.

CEPAL/ILPES (Comisión Económica para América Latina y el Caribe/Instituto Latinoamericano y del Caribe de Planificación Económica y Social) (2012), "Progresos en materia de planificación en América Latina", Los fundamentos de la planificación del desarrollo en América Latina y el Caribe: textos seleccionados del ILPES (1962-1972), Libros de la CEPAL, № 116 (LC/G.2552-P), R. Martner y J. Máttar (comps.), Santiago, Comisión Económica para América Latina y el Caribe (CEPAL), diciembre.

Chirom, J. (1985), "La profesión contable: ejercicio, antecedentes, marco legal", tesis sobre la práctica profesional, Buenos Aires.

Cuello, R. y C. Tandeciarz (1964), Programas de desarrollo económico: introducción a su estudio, Buenos Aires, Editorial Universitaria de Buenos Aires (Eudeba).

Dagnino Pastore, J. y M. Fernández López (1988), "Los economistas en el gobierno argentino", Crónicas económicas: Argentina, 1969-1988, J. Dagnino Pastore (ed.), Buenos Aires, Editorial Crespillo.

De Pablo, J. (1995), Apuntes a mitad de camino: economía sin corbata, Buenos Aires, Ediciones Macchi.

Dieulefait, C. (1958), "El modelo de insumo-producto y el problema de inversión", Revista de Ciencias Económicas, vol. 4, № 3.

Fernández Balmaceda, R. y otros (1965), "Construcción de modelos de insumo-producto en la República Argentina", Desarrollo Económico: Revista de Ciencias Sociales, vol. 5, № 17/19.

Furtado, C. (1988), La fantasía organizada, Buenos Aires, Editorial Universitaria de Buenos Aires (Eudeba).

Grupe, H. y O. Cornblit (1960), Curso intensivo de capacitación sobre problemas de desarrollo económico, Mendoza, Consejo Federal de Inversiones (CFI). 
ILPES (Instituto Latinoamericano y del Caribe de Planificación Económica y Social) (2012a), "Evolución y orientaciones del Instituto Latinoamericano de Planificación Económica y Social: nota del director general", Los fundamentos de la planificación del desarrollo en América Latina y el Caribe: textos seleccionados del ILPES (1962-1972), Libros de la CEPAL, № 116 (LC/G.2552-P), R. Martner y J. Máttar (comps.), Santiago, Comisión Económica para América Latina y el Caribe (CEPAL).

(2012b), "Evolución y perspectivas de los procesos de planificación en América Latina", Los fundamentos de la planificación del desarrollo en América Latina y el Caribe: textos seleccionados del ILPES (1962-1972), Libros de la CEPAL, № 116 (LC/G.2552-P), R. Martner y J. Máttar (comps.), Santiago, Comisión Económica para América Latina y el Caribe (CEPAL).

Jáuregui, A. (2014), "La planificación en la Argentina del desarrollo (1955-1973)", Temas de historia argentina y americana, № 22.

(2013), "La planificación en la Argentina: el CONADE y el PND (1960-1966)", Anuario del Centro de Estudios Históricos "Prof. Carlos S. A. Segreti", № 13.

Leontief, W. (1951), The Structure of American Economy, 1919-1939: An Empirical Application of Equilibrium Analysis, Nueva York, Oxford University Press.

Reig, E. (1958), "Notas universitarias: reforma del plan de estudios. La enseñanza universitaria en EE.UU.", Revista de Ciencias Económicas, vol. 4, № 2.

San Miguel, M. y otros (1961), "Examen crítico de la actual situación económica del país en el aspecto relacionado con la estabilidad monetaria y la producción", Revista de Ciencias Económicas, vol. 49, № 16.

Sunkel, O. (2000), "La labor de la CEPAL en sus primeros dos decenios", La CEPAL en sus 50 años: notas de un seminario conmemorativo, Libros de la CEPAL, № 54 (LC/G.2103-P), Santiago, Comisión Económica para América Latina y el Caribe, julio.

Toranzos, F. (1962), "Modelo lineal de expansión económica equilibrada", Revista de Ciencias Económicas, № 18.

UBA (Universidad de Buenos Aires) (1975), "Programa de la materia 315, ‘Desarrollo Económico'” [en línea] http://biblio.econ.uba.ar/cgi-bin/koha/opac-detail.pl?biblionumber=192679\&query_desc=ti\%2Cwrdll\%3A\%20 programa\%20desarrollo\%20econ\%C3\%B3mico.

(1973), "Programa de la materia 315, 'Desarrollo Económico'” [en línea] http://biblio.econ.uba.ar/cgi-bin/ koha/opac-detail.pl?biblionumber=192678\&query_desc=ti\%2Cwrdl\%3A\%20programa\%20desarrollo\%20 econ\%C3\%B3mico.

(1967), "Seminario sobre política del desarrollo económico", Buenos Aires, Facultad de Ciencias Económicas. (1965), "Curso intensivo de capacitación sobre problemas del desarrollo económico", Buenos Aires, Facultad de Ciencias Económicas.

(1964), "Curso intensivo de capacitación sobre problemas del desarrollo económico: elementos de contabilidad general, contabilidad nacional", Buenos Aires, Facultad de Ciencias Económicas.

_ (1963a), "Curso intensivo de capacitación sobre problemas del desarrollo económico", Buenos Aires, Facultad de Ciencias Económicas.

(1963b), "Programa Seminario sobre política del desarrollo económico", Buenos Aires, Facultad de Ciencias Económicas. 